\title{
An alternative formulation of finite difference WENO schemes with Lax-Wendroff time discretization for conservation laws
}

\author{
Yan Jiang ${ }^{1}$, Chi-Wang Shu ${ }^{2}$ and Mengping Zhang ${ }^{3}$
}

\begin{abstract}
We develop an alternative formulation of conservative finite difference weighted essentially non-oscillatory (WENO) schemes to solve conservation laws. In this formulation, the WENO interpolation of the solution and its derivatives are used to directly construct the numerical flux, instead of the usual practice of reconstructing the flux functions. Even though this formulation is more expensive than the standard formulation, it does have several advantages. The first advantage is that arbitrary monotone fluxes can be used in this framework, while the traditional practice of reconstructing flux functions can be applied only to smooth flux splitting. The second advantage, which is fully explored in this paper, is that it is more straightforward to construct a Lax-Wendroff time discretization procedure, with a Taylor expansion in time and with all time derivatives replaced by spatial derivatives through the partial differential equations, resulting in a narrower effective stencil compared with previous high order finite difference WENO scheme based on the reconstruction of flux functions with a Lax-Wendroff time discretization. We will describe the scheme formulation and present numerical tests for one- and two-dimensional scalar and system conservation laws demonstrating the designed high order accuracy and non-oscillatory performance of the schemes constructed in this paper.

Key Words: weighted essentially non-oscillatory scheme, Lax-Wendroff time discretization, high order accuracy

\footnotetext{
${ }^{1}$ School of Mathematical Sciences, University of Science and Technology of China, Hefei, Anhui 230026, P.R. China. E-mail: jiangy@mail.ustc.edu.cn

${ }^{2}$ Division of Applied Mathematics, Brown University, Providence, RI 02912, USA. E-mail: shu@dam.brown.edu. Research supported by AFOSR grant F49550-12-1-0399 and NSF grant DMS1112700 .

${ }^{3}$ School of Mathematical Sciences, University of Science and Technology of China, Hefei, Anhui 230026, P.R. China. E-mail: mpzhang@ustc.edu.cn. Research supported by NSFC grants 11071234, 91130016 and 91024025.
} 


\section{Report Documentation Page}

Public reporting burden for the collection of information is estimated to average 1 hour per response, including the time for reviewing instructions, searching existing data sources, gathering and maintaining the data needed, and completing and reviewing the collection of information. Send comments regarding this burden estimate or any other aspect of this collection of information, including suggestions for reducing this burden, to Washington Headquarters Services, Directorate for Information Operations and Reports, 1215 Jefferson Davis Highway, Suite 1204, Arlington VA 22202-4302. Respondents should be aware that notwithstanding any other provision of law, no person shall be subject to a penalty for failing to comply with a collection of information if it does not display a currently valid OMB control number.

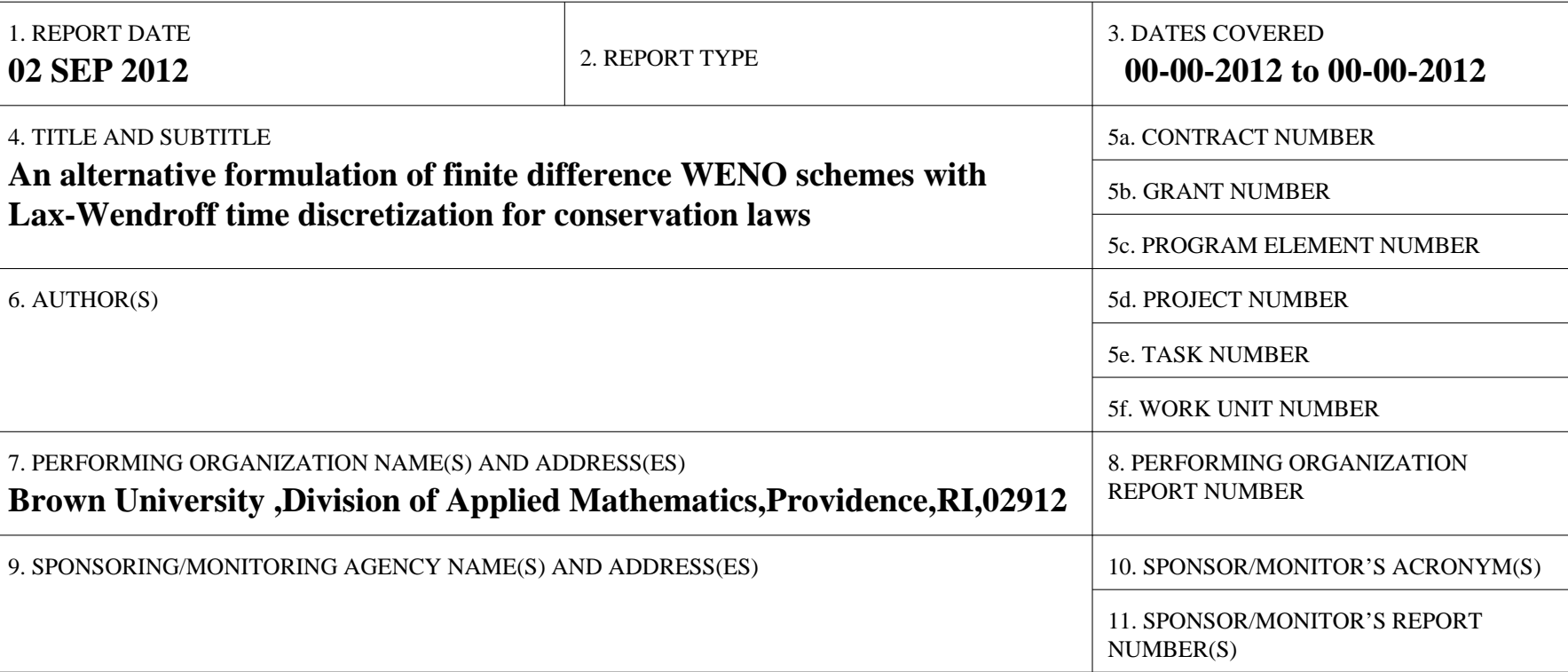

12. DISTRIBUTION/AVAILABILITY STATEMENT

Approved for public release; distribution unlimited

13. SUPPLEMENTARY NOTES

14. ABSTRACT

We develop an alternative formulation of conservative finite difference weighted essentially non-oscillatory (WENO) schemes to solve conservation laws. In this formulation the WENO interpolation of the solution and its derivatives are used to directly construct the numerical flux, instead of the usual practice of reconstructing the flux functions. Even though this formulation is more expensive than the standard formulation, it does have several advantages. The first advantage is that arbitrary monotone fluxes can be used in this framework, while the traditional practice of reconstructing flux functions can be applied only to smooth flux splitting. The second advantage, which is fully explored in this paper, is that it is more straightforward to construct a Lax-Wendroff time discretization procedure, with a Taylor expansion in time and with all time derivatives replaced by spatial derivatives through the partial differential equations, resulting in a narrower effective stencil compared with previous high order finite difference WENO scheme based on the reconstruction of flux functions with a Lax-Wendroff time discretization. We will describe the scheme formulation and present numerical tests for one- and two-dimensional scalar and system conservation laws demonstrating the designed high order accuracy and non-oscillatory performance of the schemes constructed in this paper.

15. SUBJECT TERMS

16. SECURITY CLASSIFICATION OF:

a. REPORT

unclassified b. ABSTRACT unclassified
17. LIMITATION OF ABSTRACT

Same as Report (SAR)
18. NUMBER OF PAGES

32 19a. NAME OF RESPONSIBLE PERSON unclassified 


\section{Introduction}

In this paper, we are interested in designing an alternative formulation of high order conservative finite difference WENO (weighted essentially non-oscillatory) methods, with the Lax-Wendroff time discretizations, in solving nonlinear hyperbolic conservation laws

$$
\left\{\begin{array}{l}
u_{t}+\nabla \cdot f(u)=0 \\
u(x, 0)=u_{0}(x)
\end{array}\right.
$$

On a uniform mesh $x_{i}=i \Delta x$, a semi-discrete conservative finite difference scheme for solving (1) has the following form

$$
\frac{d}{d t} u_{i}+\frac{1}{\Delta x}\left(\hat{f}_{i+\frac{1}{2}}-\hat{f}_{i-\frac{1}{2}}\right)=0
$$

where $u_{i}$ is an approximation to the point value $u\left(x_{i}, t\right)$, and the numerical flux

$$
\hat{f}_{i+\frac{1}{2}}=\hat{f}\left(u_{i-r}, \ldots, u_{i+s}\right)
$$

is designed so that

$$
\frac{1}{\Delta x}\left(\hat{f}_{i+\frac{1}{2}}-\hat{f}_{i-\frac{1}{2}}\right)=\left.f(u(x))_{x}\right|_{x_{i}}+O\left(\Delta x^{k}\right)
$$

for a $k$-th order scheme. Most of the high order finite difference schemes, for example the high order finite difference WENO schemes in $[4,1,12]$, use the following simple Lemma in [14]:

Lemma 1.1 (Shu and Osher [14]): If a function $h(x)$ satisfies the following relationship

$$
f(u(x))=\frac{1}{\Delta x} \int_{x-\frac{\Delta x}{2}}^{x+\frac{\Delta x}{2}} h(\xi) d \xi
$$

then

$$
\frac{1}{\Delta x}\left(h\left(x+\frac{\Delta x}{2}\right)-h\left(x-\frac{\Delta x}{2}\right)\right)=f(u(x))_{x} .
$$

The proof of this lemma is a simple application of the fundamental theorem of calculus. 
With this simple lemma, it is clear that we should take the numerical flux as

$$
\hat{f}_{i+\frac{1}{2}}=h\left(x_{i+\frac{1}{2}}\right)+O\left(\Delta x^{k}\right)
$$

with a smooth coefficient in the $O\left(\Delta x^{k}\right)$ term, to obtain the high order accuracy (3). Since the definition of the function $h(x)$ in (4) implies

$$
\bar{h}_{i}=\frac{1}{\Delta x} \int_{x_{i-\frac{1}{2}}}^{x_{i+\frac{1}{2}}} h(\xi) d \xi=f\left(u_{i}\right)
$$

which is known once we have the point values $u_{i}$, we are facing the standard reconstruction problem of knowing the cell averages $\left\{\bar{h}_{i}\right\}$ of the function $h(x)$, and seeking to reconstruct its point values at the cell boundaries $h\left(x_{i+\frac{1}{2}}\right)$ to high order accuracy, to be used as the numerical flux $\hat{f}_{i+\frac{1}{2}}$ in (6). This reconstruction problem is the backbone of the standard finite volume schemes, for example the essentially non-oscillatory (ENO) finite volume schemes [3] and WENO finite volume schemes [7]. Therefore, using the simple Lemma 1.1, we can simply apply the standard reconstruction subroutine in a high order finite volume scheme to obtain the numerical flux $\left\{\hat{f}_{i+\frac{1}{2}}\right\}$ for a high order conservative finite difference scheme from the flux point values $\left\{f\left(u_{i}\right)\right\}$.

Because of the clean conception and easy implementation, this formulation of numerical fluxes in high order conservative finite difference schemes has been used widely, for example in high order finite difference ENO schemes [14] and WENO schemes [4, 1, 12]. However, there are several disadvantages of this formulation:

1. In order to achieve upwinding and nonlinear stability, an approximate Riemann solver or a two-point monotone flux is used in finite volume schemes. With this finite difference flux formulation, upwinding can be implemented easily only for the following smooth flux splitting

$$
f(u)=f^{+}(u)+f^{-}(u)
$$

where $\frac{d}{d u} f^{+}(u) \geq 0$ (or in system case, this Jacobian matrix has only real and positive eigenvalues) and $\frac{d}{d u} f^{-}(u) \leq 0$ (or in system case, this Jacobian matrix has 
only real and negative eigenvalues). In order to guarantee high order accuracy, the two functions $f^{+}(u)$ and $f^{-}(u)$ should be as smooth functions of $u$ as $f(u)$. The most common flux splitting used in finite difference schemes is therefore the LaxFriedrichs flux splitting, which is the most diffusive among two-point monotone fluxes.

2. The Lax-Wendroff time discretization procedure [8] is difficult to design and results in a rather wide effective stencil. We will elaborate in more details on this point later in the paper.

3. Since the reconstruction is performed directly on the flux values $\left\{f\left(u_{i}\right)\right\}$ (or $\left\{f^{+}\left(u_{i}\right)\right\}$ and $\left\{f^{-}\left(u_{i}\right)\right\}$ with flux splitting), not on the point values of the solution $\left\{u_{i}\right\}$, it is more difficult to maintain free stream solutions exactly in curvilinear meshes for multi-dimensional flow computation. This is because the fluxes in curvilinear coordinates involve metric derivatives, resulting in non-exact cancellations when nonlinear reconstructions are performed for different fluxes.

We would like to explore an alternative formulation for constructing numerical fluxes in high order conservative finite difference schemes, originally designed in [13], which involves interpolations directly on the point values of the solution $\left\{u_{i}\right\}$ rather than on the flux values. This alternative formulation, even though less clean and more computationally expensive, does overcome all three disadvantages listed above. We will explore the overcoming of the first two disadvantages, especially the second one, in detail in this paper.

We now give a brief survey of WENO schemes and Lax-Wendroff time discretization. WENO schemes are high order schemes for approximating hyperbolic conservation laws and other convection dominated partial differential equations. They can produce sharp, non-oscillatory discontinuity transitions and high order accurate resolutions for the smooth part of the solution. The first WENO scheme was introduced in 1994 by 
Liu, Osher and Chan in their pioneering paper [7], in which a third order accurate finite volume WENO scheme in one space dimension was constructed. In [4], a general framework is provided to design arbitrary high order accurate finite difference WENO schemes, which are more efficient for multi-dimension calculations. Very high order finite difference WENO schemes are documented in [1].

The Lax-Wendroff time discretization procedure, which is also called the Taylor type time discretization, is based on the idea of the classical Lax-Wendroff scheme [5], relying on writing the solution at the next time step by Taylor expansion in time, and repeatedly using the partial differential equation (PDE) to rewrite the time derivatives in this Taylor expansion as spatial derivatives. These spatial derivatives can then be discretized by standard approximation procedures, for example the WENO approximation. We denote $u_{i}^{n}$ as the approximation of the point values $u\left(x_{i}, t^{n}\right)$, and $u^{(r)}$ as the $r$-th order time derivative of $u$, namely $u^{(r)}=\frac{\partial^{r} u}{\partial t^{r}}$. We also use $u^{\prime}, u^{\prime \prime}$ and $u^{\prime \prime \prime}$ to denote the first three time derivatives of $u$. By Taylor expansion, we obtain

$$
u(x, t+\Delta t)=u(x, t)+\Delta t u^{\prime}+\frac{\Delta t^{2}}{2 !} u^{\prime \prime}+\frac{\Delta t^{3}}{3 !} u^{\prime \prime \prime}+\frac{\Delta t^{4}}{4 !} u^{(4)}+\frac{\Delta t^{5}}{5 !} u^{(5)}+\cdots .
$$

If we approximate the first $k$ derivatives, we can get $k$-th order accuracy in time. The main idea of the Lax-Wendroff time discretization procedure is to rewrite the time derivatives $u^{(r)}$ as spatial derivatives utilizing the PDE, then discretize these spatial derivatives to sufficient accuracy. This is rather straightforward for a finite volume scheme. However, for a finite difference scheme using the formulation (6) and Lemma 1.1 to compute its numerical fluxes, it is more difficult to implement the Lax-Wendroff procedure because we do not have approximations to the spatial derivatives of the solution $u$ at our disposal, only approximations to the spatial derivatives of the flux function $f(u)$. In [8], Qiu and Shu designed a finite difference WENO scheme with Lax-Wendroff time discretization using the formulation (6) and Lemma 1.1. To avoid the difficulty mentioned above, they use the approximations to the lower order time derivatives to approximate the higher order ones. As a consequence, the method in [8] involves a rather wide effective stencil. 
We will provide more details to illustrate this phenomenon. In the procedure to build a fifth order in space and fourth order in time scheme in [8] for solving the one-dimensional scalar equation $u_{t}+f(u)_{x}=0$, the following steps are involved:

Step 1. Using the PDE, we obtain $u^{\prime}=-f(u)_{x}$, and the first order time derivative can be obtained by the conservative finite difference WENO approximation (3). To obtain a fifth order WENO approximation for $\left.u^{\prime}\right|_{j} ^{n}$, we would need the point values $S=\left\{u_{j-3}^{n}, \cdots, u_{j+3}^{n}\right\}$.

Step 2. When constructing the second order time derivative $u^{\prime \prime}=-\left(f^{\prime}(u) u^{\prime}\right)_{x}$, we denote $g_{j}=f^{\prime}\left(u_{j}\right) u_{j}^{\prime}$, where $u_{j}^{\prime}$ are the point values of the first order time derivative computed in the previous Step 1. Then we can use a fourth order central difference formula to approximate $u^{\prime \prime}=-g_{x}$ at the point $\left(x_{j}, t^{n}\right)$ :

$$
u_{j}^{\prime \prime}=-\frac{1}{12 \Delta x}\left(g_{j-2}-8 g_{j-1}+8 g_{j+1}-g_{j+2}\right) .
$$

It was observed in [8] that the more costly WENO approximation is not necessary for second and higher order time derivatives, as the standard central difference approximations perform well. Considering $g_{j}=f^{\prime}\left(u_{j}\right) u_{j}^{\prime}$ and using Step 1 to obtain $u_{j}^{\prime}$, we have the effective stencil for obtaining the point value $\left.u^{\prime \prime}\right|_{j} ^{n}$ to be $S=\left\{u_{j-5}^{n}, \cdots, u_{j+5}^{n}\right\}$.

Step 3. When constructing the third order time derivative $u^{\prime \prime \prime}=-\left(f^{\prime}(u) u^{\prime \prime}+f^{\prime \prime}(u)\left(u^{\prime}\right)^{2}\right)_{x}$, we let $g_{j}=\left(f^{\prime}\left(u_{j}\right) u_{j}^{\prime \prime}+f^{\prime \prime}\left(u_{j}\right)\left(u^{\prime}\right)_{j}^{2}\right)$, where the point values $u_{j}^{\prime}$ and $u_{j}^{\prime \prime}$ are obtained in the previous Step 1 and Step 2, respectively. Then we repeat Step 2 to approximate the third order time derivative using a fourth order central difference approximation (third order accuracy is enough here, but central approximations provide only even orders of accuracy). Considering $u_{j}^{\prime}$ and $u_{j}^{\prime \prime}$ are obtained in Step 1 and Step 2, we obtain the effective stencil for approximating $u^{\prime \prime \prime}$ at the point $\left(x_{j}, t^{n}\right)$ to be $S=\left\{u_{j-7}^{n}, \cdots, u_{j+7}^{n}\right\}$.

Step 4. The construction of the fourth order time derivative $u^{(4)}=-\left(f^{\prime}(u) u^{\prime \prime \prime}+\right.$ $\left.3 f^{\prime \prime}(u) u^{\prime} u^{\prime \prime}+f^{\prime \prime \prime}(u)\left(u^{\prime}\right)^{3}\right)_{x}$ is obtained in a similar fashion. Let $g_{j}=f^{\prime}\left(u_{j}\right) u_{j}^{\prime \prime \prime}+3 f^{\prime \prime}\left(u_{j}\right) u_{j}^{\prime} u_{j}^{\prime \prime}+$ 
$f^{\prime \prime \prime}\left(u_{j}\right)\left(u^{\prime}\right)_{j}^{3}$, where the point values $u_{j}^{\prime}, u_{j}^{\prime \prime}$ and $u_{j}^{\prime \prime \prime}$ are obtained in the previous Steps 1, 2 and 3 respectively. Then we use a second order central difference to approximate $u_{j}^{(4)}=-\frac{1}{2 \Delta x}\left(g_{j+1}-g_{j-1}\right)$. Thus, to get the value $u^{(4)}$ at the point $\left(x_{j}, t^{n}\right)$, we actually need point values in the effective stencil $S=\left\{u_{j-8}^{n}, \cdots, u_{j+8}^{n}\right\}$.

In summary, to obtain a fifth order in space and fourth order in time Lax-Wendroff type WENO scheme in [8], the effective stencil is $S=\left\{I_{j-8}, \cdots, I_{j+8}\right\}$ for the point $x_{j}$, containing 17 grid points. This is a pretty wide stencil. Of course, it is still narrower than Runge-Kutta time discretization. When a four-stage, fourth order Runge-Kutta

time discretization is used on a fifth order finite difference WENO scheme, the width of the effective stencil is 25 grid points.

One major motivation to discuss the alternative formulation of high order conservative finite difference schemes in this paper is to make the effective stencil narrower when applying the Lax-Wendroff time discretization. We will review WENO interpolation in Section 2, which is the main approximation tool for the alternative formulation of high order conservative finite difference schemes. Details on the construction and implementation of the alternative formulation will be described in Section 3, for oneand two-dimensional scalar and system equations. In Section 4, extensive numerical examples are provided to demonstrate the accuracy and effectiveness of the methods. Concluding remarks are given in Section 5 .

\section{WENO interpolation}

A major building block of the alternative formulation of high order conservative finite difference scheme discussed in this paper is the following WENO interpolation procedure. Given the point values $u_{i}=u\left(x_{i}\right)$ of a piecewise smooth function $u(x)$, we would like to find an approximation of $u(x)$ at the half nodes $x_{i+\frac{1}{2}}$. This WENO interpolation procedure has been developed in, e.g. $[10,2,12]$ and will be described briefly below.

The first component of the general WENO produce is a set of lower order approxi- 
mations to the target value, based on different stencils, which are referred to as the small stencils. We would find a unique polynomial of degree at most $k-1$, denoted by $p_{r}(x)$, which interpolates the function $u(x)$, namely $p_{r}\left(x_{j}\right)=u_{j}$, at the mesh points $x_{j}$ in the small stencil $S_{r}=\left\{x_{i-r}, x_{i-r+1}, \ldots, x_{i+s}\right\}$ for $r=0,1, \ldots, k-1$, with $r+s+1=k$. Then, we would use $u_{i+\frac{1}{2}}^{(r)}=p_{r}\left(x_{i+\frac{1}{2}}\right)$ as an approximation to the value $u\left(x_{i+\frac{1}{2}}\right)$, and we have

$$
u_{i+\frac{1}{2}}^{(r)}-u\left(x_{i+\frac{1}{2}}\right)=O\left(\Delta x^{k}\right)
$$

if the function $u(x)$ is smooth in the stencil $S_{r}$. For example, when $k=3$, we have $S_{0}=\left\{x_{i}, x_{i+1}, x_{i+2}\right\}, S_{1}=\left\{x_{i-1}, x_{i}, x_{i+1}\right\}, S_{2}=\left\{x_{i-2}, x_{i-1}, x_{i}\right\}$, and

$$
\begin{aligned}
& u_{i+\frac{1}{2}}^{(0)}=\frac{3}{8} u_{i}+\frac{3}{4} u_{i+1}-\frac{1}{8} u_{i+2}, \\
& u_{i+\frac{1}{2}}^{(1)}=-\frac{1}{8} u_{i-1}+\frac{3}{4} u_{i}+\frac{3}{8} u_{i+1}, \\
& u_{i+\frac{1}{2}}^{(2)}=\frac{3}{8} u_{i-2}-\frac{5}{4} u_{i-1}+\frac{15}{8} u_{i} .
\end{aligned}
$$

The second component of the general WENO procedure is to combine the set of lower order approximations to form a higher order approximation to the same target value, based on the big stencil $S=\left\{x_{i-k+1}, \ldots, x_{i+k-1}\right\}$, which is the union of all the small stencils in the first component. We would find a unique polynomial of degree at most $2 k-2$, denoted by $p(x)$, interpolating the function $u(x)$ at the points in the big stencil. We use $u_{i+\frac{1}{2}}=p\left(x_{i+\frac{1}{2}}\right)$ as the approximation to the value $u\left(x_{i+\frac{1}{2}}\right)$, and we have

$$
u_{i+\frac{1}{2}}-u\left(x_{i+\frac{1}{2}}\right)=O\left(\Delta x^{2 k-1}\right)
$$

provided that the function $u(x)$ is smooth in the big stencil $S$. When $k=3$, we have

$$
u_{i+\frac{1}{2}}=\frac{3}{128} u_{i-2}-\frac{5}{32} u_{i-1}+\frac{45}{64} u_{i}+\frac{15}{32} u_{i+1}-\frac{5}{128} u_{i+2} .
$$

There are constants $d_{r}$ such that

$$
u_{i+\frac{1}{2}}=\sum_{r=0}^{k-1} d_{r} u_{i+\frac{1}{2}}^{(r)}
$$


where the constants satisfy $\sum_{r=0}^{k-1} d_{r}=1$. These constants are usually referred as the linear weights in the WENO procedure. For the case $k=3$, the linear weights are given as

$$
d_{0}=\frac{5}{16}, \quad d_{1}=\frac{5}{8}, \quad d_{2}=\frac{1}{16} .
$$

The third component of the general WENO procedure is to change the linear weights into nonlinear weights, to ensure both accuracy in smooth cases and non-oscillatory performance when at least one of the small stencils contain a discontinuity of the function $u(x)$. We can achieve this through changing the linear weights $d_{r}$ into the nonlinear weights $\omega_{r}$, defined by

$$
\omega_{r}=\frac{\alpha_{r}}{\sum_{s=0}^{k-1} \alpha_{s}}, \quad r=0, \ldots, k-1
$$

with

$$
\alpha_{r}=\frac{d_{r}}{\left(\epsilon+\beta_{r}\right)^{2}}
$$

Here, we take $\epsilon=10^{-6}$, avoiding the denominator to be zero, and $\beta_{r}$ are the so-called smoothness indicators of the stencil $S_{r}$, which measures the smoothness of $u(x)$ in this stencil. Following the original WENO recipe [4], we define

$$
\beta_{r}=\sum_{l=1}^{k-1} \int_{x_{i-\frac{1}{2}}}^{x_{i+\frac{1}{2}}} \Delta x^{2 l-1}\left(\frac{\partial^{l} p_{r}(x)}{\partial^{l} x}\right)^{2} d x .
$$

When $k=3$, the formula gives

$$
\begin{aligned}
& \beta_{0}=\frac{13}{12}\left(u_{i}-2 u_{i+1}+u_{i+2}\right)^{2}+\frac{1}{4}\left(3 u_{i}-4 u_{i+1}+u_{i+2}\right)^{2}, \\
& \beta_{1}=\frac{13}{12}\left(u_{i-1}-2 u_{i}+u_{i+1}\right)^{2}+\frac{1}{4}\left(u_{i-1}-u_{i+1}\right)^{2}, \\
& \beta_{2}=\frac{13}{12}\left(u_{i-2}-2 u_{i-1}+u_{i}\right)^{2}+\frac{1}{4}\left(u_{i-2}-4 u_{i-1}+3 u_{i}\right)^{2} .
\end{aligned}
$$

Thus, we get the WENO interpolation of $u(x)$ at the point $x_{i+\frac{1}{2}}$ as

$$
u_{i+\frac{1}{2}}=\sum_{r=0}^{k-1} \omega_{r} u_{i+\frac{1}{2}}^{(r)} .
$$


We denote it by $u_{i+\frac{1}{2}}^{-}$since the big stencil $S$ used to obtain this approximation is biased to the left (there is one more grid point to the left of $x_{i+\frac{1}{2}}$ than to the right). If $u(x)$ is smooth in this big stencil $S$, we have

$$
u_{i+\frac{1}{2}}^{-}-u\left(x_{i+\frac{1}{2}}\right)=O\left(\Delta x^{2 k-1}\right)
$$

If one of the small stencils contains a discontinuity, the corresponding smoothness indicator will be much larger than that of other small stencils, hence the nonlinear weight corresponding to this small stencil will be very small, thus achieving essentially non-oscillatory performance. If we choose the big stencil as $S=\left\{x_{i-k+2}, \ldots, x_{i+k}\right\}$ which is biased to the right, and the small stencils as $S_{r}=\left\{x_{i-r+1}, \ldots, x_{i-r+k}\right\}$, for $r=0, \ldots, k-1$, we can obtain in a similar fashion a WENO interpolation of $u(x)$ at the point $x_{i+\frac{1}{2}}$, denoted by $u_{i+\frac{1}{2}}^{+}$, which has the same high order accuracy in smooth regions and essentially nonoscillatory performance near discontinuities as $u_{i+\frac{1}{2}}^{-}$. In fact, the process to obtain $u_{i+\frac{1}{2}}^{+}$ is mirror-symmetric to that for $u_{i+\frac{1}{2}}^{-}$, with respect to the target point $x_{i+\frac{1}{2}}$.

\section{Construction of the scheme}

\subsection{Construction of the numerical flux in one-dimension}

Assuming that $f(u)$ is a smooth function of $u$, we would like to find a consistent numerical flux function

$$
\hat{f}_{i+\frac{1}{2}}=\hat{f}\left(u_{i-r}, \ldots, u_{i+s}\right)
$$

such that the flux difference approximates the derivative $f(u(x))_{x}$ to $k$-th order accuracy

$$
\frac{1}{\Delta x}\left(\hat{f}_{i+\frac{1}{2}}-\hat{f}_{i-\frac{1}{2}}\right)=\left.f(u(x))_{x}\right|_{x_{i}}+O\left(\Delta x^{k}\right)
$$

The numerical flux should satisfy the following conditions:

- $\hat{f}$ is a Lipschitz continuous function in all the arguments;

- $\hat{f}$ is consistent with the physical flux $f$, that is, $\hat{f}(u, \cdots, u)=f(u)$. 
It has been shown in [13] that there exist constants $a_{2}, a_{4}, \ldots$ such that

$$
\hat{f}_{i+\frac{1}{2}}=f_{i+\frac{1}{2}}+\sum_{k=1}^{[(r-1) / 2]} a_{2 k} \Delta x^{2 k}\left(\frac{\partial^{2 k}}{\partial x^{2 k}} f\right)_{i+\frac{1}{2}}+O\left(\Delta x^{r+1}\right)
$$

which guarantees $k=r$-th order accuracy in (16). The coefficients $a_{2 r}$ in (17) can be obtained through Taylor expansion and the accuracy constraint (16). To get an approximation with fifth order accuracy $(k=5$ in $(16))$, we can use the first three terms given as

$$
\hat{f}_{i+\frac{1}{2}}=f_{i+\frac{1}{2}}-\left.\frac{1}{24} \Delta x^{2} f_{x x}\right|_{i+\frac{1}{2}}+\left.\frac{7}{5760} \Delta x^{4} f_{x x x x}\right|_{i+\frac{1}{2}}
$$

The first term of the numerical flux in (18) is approximated by

$$
f_{i+\frac{1}{2}}=h\left(u_{i+\frac{1}{2}}^{-}, u_{i+\frac{1}{2}}^{+}\right)
$$

with the values $u_{i+\frac{1}{2}}^{ \pm}$obtained by the WENO interpolation discussed in the previous section. The two-argument function $h$ is a monotone flux. It satisfies:

- $h(a, b)$ is a Lipschitz continuous function in both argument;

- $h(a, b)$ is a nondecreasing function in $a$ and a nonincreasing function in $b$. Symbolically $h(\uparrow, \downarrow)$;

- $h(a, b)$ is consistent with the physical flux $f$, that is, $h(a, a)=f(a)$.

Examples of the monotone fluxes include:

1. The Godunov flux:

$$
h(a, b)= \begin{cases}\min _{a \leq x \leq b} f(u), & \text { if } a \leq b \\ \max _{b \leq x \leq a} f(u), & \text { if } a>b\end{cases}
$$

2. The Engquist-Osher flux:

$$
h(a, b)=\int_{0}^{a} \max \left(f^{\prime}(u), 0\right) d u+\int_{0}^{b} \min \left(f^{\prime}(u), 0\right) d u+f(0)
$$


3. The Lax-Friedrichs flux:

$$
h(a, b)=\frac{1}{2}[f(a)+f(b)-\alpha(b-a)]
$$

where $\alpha=\max _{u}\left|f^{\prime}(u)\right|$ is a constant. The maximum is taken over the relevant range of $u$.

More discussions on monotone fluxes can be found in, e.g. [6].

The remaining terms of the numerical flux in (18) have at least $\Delta x^{2}$ in their coefficients, hence they only need lower order approximations and they are expected to contribute much less to spurious oscillations. Therefore, following the practice in [8], we approximate these remaining terms by simple central approximation or one-point upwind-biased approximation with suitable orders of accuracy, without using the more expensive WENO procedure.

\subsection{One-dimensional scalar case}

Consider the one-dimension scalar conservation law

$$
\left\{\begin{array}{l}
u_{t}+f(u)_{x}=0 \\
u(x, 0)=u_{0}(x)
\end{array}\right.
$$

We describe the Lax-Wendroff time discretization to get $k$-th order accuracy in time. In this paper, we will implement the procedure up to fourth order in time.

1. The approximation of the first time derivative $u^{\prime}=-f(u)_{x}$ follows the standard WENO procedure. We construct the numerical flux with $(2 r-1)$-th order accuracy as described in the previous section. In particular, the points $\left\{x_{i-r+1}, \ldots, x_{i+r}\right\}$ are required to obtain the WENO interpolation $u_{i+\frac{1}{2}}^{ \pm}$. For the spatial derivatives $\frac{\partial^{k} f}{\partial x^{k}}$ at $x_{i+\frac{1}{2}}$, we use $(2 r-1-k)$-th order simple finite difference approximations based on the same points $\left\{x_{i-r+1}, \ldots, x_{i+r}\right\}$. In our numerical experiments, we aim for fifth order spatial accuracy and take $r=3$. The numerical flux corresponding 
to $u^{\prime}$ is then given as

$$
\begin{aligned}
\hat{f}_{i+\frac{1}{2}}^{(1)} & =h\left(u_{i+\frac{1}{2}}^{-}, u_{i+\frac{1}{2}}^{+}\right)-\left.\frac{1}{24} \Delta x^{2}\left(f^{\prime \prime}(u)\left(u_{x}\right)^{2}+f^{\prime}(u) u_{x x}\right)\right|_{x_{i+\frac{1}{2}}}+\frac{7}{5760} \Delta x^{4}\left(f^{\prime \prime \prime \prime}(u)\left(u_{x}\right)^{4}\right. \\
& \left.+6 f^{\prime \prime \prime}(u)\left(u_{x}\right)^{2} u_{x x}+4 f^{\prime \prime}(u) u_{x} u_{x x x}+3 f^{\prime \prime}(u)\left(u_{x x}\right)^{2}+f^{\prime}(u) u_{x x x x}\right)\left.\right|_{x_{i+\frac{1}{2}}}
\end{aligned}
$$

such that

$$
\frac{\hat{f}_{i+\frac{1}{2}}^{(1)}-\hat{f}_{i-\frac{1}{2}}^{(1)}}{\Delta x}=-\left.u^{\prime}\right|_{x_{i}}+O\left(\Delta x^{4}\right)
$$

where $u_{i+\frac{1}{2}}^{ \pm}$in the $h\left(u_{i+\frac{1}{2}}^{-}, u_{i+\frac{1}{2}}^{+}\right)$term are obtained through the fifth order WENO interpolation, and the values of $u, u_{x}, u_{x x}, u_{x x x}$ and $u_{x x x x}$ at $x_{i+\frac{1}{2}}$ in the remaining terms are obtained from the same polynomial interpolation based on the points $\left\{x_{i-2}, \ldots, x_{i+3}\right\}$

2. The next step is to obtain the approximation of the second time derivative $u^{\prime \prime}=$ $-\left(f^{\prime}(u) u^{\prime}\right)_{x}=\left(\left(f^{\prime}(u)\right)^{2} u_{x}\right)_{x}$. We let $g=-\left(f^{\prime}(u)\right)^{2} u_{x}$, and repeat the process in Step 1. The two major differences from Step 1 are: (i) the approximation can be one order lower in accuracy since there is an extra $\Delta t$ in the coefficient; and (ii) there is no need to involve WENO in the approximation, also because of the extra $\Delta t$ in the coefficient. Therefore, we would still only need the interpolation polynomial based on the points $\left\{x_{i-2}, \ldots, x_{i+3}\right\}$ for all the values of $u, u_{x}, u_{x x}$, $u_{x x x}$ and $u_{x x x x}$ at $x_{i+\frac{1}{2}}$ in the following expression

$$
\begin{aligned}
\hat{f}_{i+\frac{1}{2}}^{(2)}=\left.g\right|_{x_{i+\frac{1}{2}}}-\left.\frac{\Delta x^{2}}{24} g_{x x}\right|_{x_{i+\frac{1}{2}}} & \\
=- & \left.\left(f^{\prime}(u)\right)^{2} u_{x}\right|_{x_{i+\frac{1}{2}}}+\frac{\Delta x^{2}}{24}\left(2\left(f^{\prime \prime}(u)\right)^{2}\left(u_{x}\right)^{3}+2 f^{\prime \prime \prime}(u) f^{\prime}(u)\left(u_{x}\right)^{3}\right. \\
& \left.+6 f^{\prime \prime}(u) f^{\prime}(u) u_{x} u_{x x}+\left(f^{\prime}(u)\right)^{2} u_{x x x}\right)\left.\right|_{x_{i+\frac{1}{2}}}
\end{aligned}
$$

such that

$$
\frac{\hat{f}_{i+\frac{1}{2}}^{(2)}-\hat{f}_{i-\frac{1}{2}}^{(2)}}{\Delta x}=-\left.u^{\prime \prime}\right|_{x_{i}}+O\left(\Delta x^{4}\right)
$$


3. The approximation of the third time derivative $u^{\prime \prime \prime}=-\left(3 f^{\prime \prime}(u)\left(f^{\prime}(u)\right)^{2} u_{x}^{2}\right.$ $\left.+\left(f^{\prime}(u)\right)^{3} u_{x x}\right)_{x}$ is obtained in a similar fashion. Let $g=\left(3 f^{\prime \prime}(u)\left(f^{\prime}(u)\right)^{2}\right.$ $\left.u_{x}^{2}+\left(f^{\prime}(u)\right)^{3} u_{x x}\right)$, we can obtain the numerical flux as

$$
\begin{aligned}
\hat{f}_{i+\frac{1}{2}}^{(3)}= & \left.g\right|_{x_{i+\frac{1}{2}}}-\left.\frac{\Delta x^{2}}{24} g_{x x}\right|_{x_{i+\frac{1}{2}}} \\
= & \left.\left(3 f^{\prime \prime}(u)\left(f^{\prime}(u)\right)^{2}\left(u_{x}\right)^{2}+\left(f^{\prime}(u)\right)^{3} u_{x x}\right)\right|_{x_{i+\frac{1}{2}}}-\frac{\Delta x^{2}}{24}\left(3 f^{\prime \prime \prime \prime}(u)\left(f^{\prime}(u)\right)^{2}\left(u_{x}\right)^{4}\right. \\
& +18 f^{\prime \prime \prime}(u) f^{\prime \prime}(u) f^{\prime}(u)\left(u_{x}\right)^{4}+18 f^{\prime \prime \prime}(u)\left(f^{\prime}(u)\right)^{2}\left(u_{x}\right)^{2} u_{x x}+6\left(f^{\prime \prime}(u)\right)^{3}\left(u_{x}\right)^{4} \\
& +36\left(f^{\prime \prime}(u)\right)^{2} f^{\prime}(u)\left(u_{x}\right)^{2} u_{x x}+12 f^{\prime \prime}(u)\left(f^{\prime}(u)\right)^{2} u_{x} u_{x x x}+9 f^{\prime \prime}(u)\left(f^{\prime}(u)\right)^{2}\left(u_{x x}\right)^{2} \\
& \left.+\left(f^{\prime}(u)\right)^{3} u_{x x x x}\right)\left.\right|_{x_{i+\frac{1}{2}}}
\end{aligned}
$$

where the values of $u, u_{x}, u_{x x}, u_{x x x}$ and $u_{x x x x}$ at $x_{i+\frac{1}{2}}$ can all be obtained from the same interpolation polynomial based on the points $\left\{x_{i-2}, \ldots, x_{i+3}\right\}$. We will then have

$$
\frac{\hat{f}_{i+\frac{1}{2}}^{(3)}-\hat{f}_{i-\frac{1}{2}}^{(3)}}{\Delta x}=-\left.u^{\prime \prime \prime}\right|_{x_{i}}+O\left(\Delta x^{3}\right)
$$

4. The final step is the approximation of the fourth time derivative $u^{(4)}=\left(4 f^{\prime \prime \prime}(u)\left(f^{\prime}(u)\right)^{3}\right.$ $\left.\left(u_{x}\right)^{3}+12\left(f^{\prime \prime}(u)\right)^{2}\left(f^{\prime}(u)\right)^{2}\left(u_{x}\right)^{3}+12 f^{\prime \prime}(u)\left(f^{\prime}(u)\right)^{3} u_{x} u_{x x x}+\left(f^{\prime}(u)\right)^{4} u_{x x x}\right)_{x}$. Let $g=$ $-\left(4 f^{\prime \prime \prime}(u)\left(f^{\prime}(u)\right)^{3}\left(u_{x}\right)^{3}+12\left(f^{\prime \prime}(u)\right)^{2}\left(f^{\prime}(u)\right)^{2}\left(u_{x}\right)^{3}+12 f^{\prime \prime}(u)\left(f^{\prime}(u)\right)^{3} u_{x} u_{x x x}+\left(f^{\prime}(u)\right)^{4} u_{x x x}\right)$, we can obtain the numerical flux as

$$
\begin{aligned}
\hat{f}_{i+\frac{1}{2}}^{(4)}= & \left.g\right|_{x_{i+\frac{1}{2}}} \\
= & -\left(4 f^{\prime \prime \prime}(u)\left(f^{\prime}(u)\right)^{3}\left(u_{x}\right)^{3}+12\left(f^{\prime \prime}(u)\right)^{2}\left(f^{\prime}(u)\right)^{2}\left(u_{x}\right)^{3}+12 f^{\prime \prime}(u)\left(f^{\prime}(u)\right)^{3} u_{x} u_{x x x}\right. \\
& \left.+\left(f^{\prime}(u)\right)^{4} u_{x x x}\right)\left.\right|_{x_{i+\frac{1}{2}}}
\end{aligned}
$$

where the values of $u, u_{x}, u_{x x}, u_{x x x}$ and $u_{x x x x}$ at $x_{i+\frac{1}{2}}$ can all be obtained from the same interpolation polynomial based on the points $\left\{x_{i-2}, \ldots, x_{i+3}\right\}$. We will then have

$$
\frac{\hat{f}_{i+\frac{1}{2}}^{(4)}-\hat{f}_{i-\frac{1}{2}}^{(4)}}{\Delta x}=-\left.u^{(4)}\right|_{x_{i}}+O\left(\Delta x^{2}\right) .
$$


5. We can form the numerical flux as the sum of all the fluxes above

$$
\hat{f}_{i+\frac{1}{2}}=\hat{f}_{i+\frac{1}{2}}^{(1)}+\frac{\Delta t}{2} \hat{f}_{i+\frac{1}{2}}^{(2)}+\frac{\Delta t^{2}}{6} \hat{f}_{i+\frac{1}{2}}^{(3)}+\frac{\Delta t^{3}}{24} \hat{f}_{i+\frac{1}{2}}^{(4)}
$$

and obtain the final scheme

$$
u_{i}^{n+1}=u_{i}^{n}-\frac{\Delta t}{\Delta x}\left(\hat{f}_{i+\frac{1}{2}}-\hat{f}_{i-\frac{1}{2}}\right)
$$

which is fifth order accurate in space and fourth order accurate in time.

Of course, we can also increase the time accuracy to fifth order, however heavier algebra will be involved. In practice, the time accuracy can usually be lower than spatial accuracy to achieve the desired resolution on modest meshes. This is also verified in our numerical results in Section 4.

Clearly, the fifth order in space, fourth order in time Lax-Wendroff finite difference scheme constructed above has an effective stencil of only 7 points, the same as the semidiscrete scheme. This compares quite favorably with the Lax-Wendroff type scheme in [8] which has an effective stencil of 17 points, or a fourth order Runge-Kutta finite difference WENO scheme which has an effective stencil of 25 points.

\subsection{One-dimensional systems}

For a one-dimensional system of conservation laws (23),

$$
u(x, t)=\left(u^{1}(x, t), \cdots, u^{m}(x, t)\right)^{T}
$$

is a vector and

$$
f(u)=\left(f^{1}\left(u^{1}, \cdots, u^{m}\right), \cdots, f^{m}\left(u^{1}, \cdots, u^{m}\right)\right)^{T}
$$

is a vector function of $u$. Suppose the system is hyperbolic, i.e. the Jacobian $f^{\prime}(u)$ has $m$ real eigenvalues

$$
\lambda_{1}(u) \leq \ldots \leq \lambda_{m}(u)
$$


and a complete set of independent eigenvectors

$$
r_{1}(u), \ldots, r_{m}(u)
$$

We denote the matrix whose columns are eigenvectors by

$$
R(u)=\left(r_{1}(u), \ldots, r_{m}(u)\right)
$$

Then

$$
R^{-1}(u) f^{\prime}(u) R(u)=\Lambda(u)
$$

where $\Lambda(u)$ is the diagonal matrix with $\lambda_{1}(u), \ldots, \lambda_{m}(u)$ on the diagonal.

Similar to the scale case, we replace the time derivatives by spatial derivatives using the PDE. We still use the numerical flux for each component

$$
\hat{g}_{i+\frac{1}{2}}=g_{i+\frac{1}{2}}+\left.\sum_{k=1}^{[(r-1) / 2]} a_{2 k} \Delta x^{2 k} \frac{\partial^{2 k} g}{\partial x^{2 k}}\right|_{i+\frac{1}{2}}+O\left(\Delta x^{r+1}\right)
$$

when approaching $g_{x}$.

When constructing the first part of numerical flux for $u_{t}$, i.e. $h\left(u_{i+\frac{1}{2}}^{-}, u_{i+\frac{1}{2}}^{+}\right)$in $\hat{f}_{i+\frac{1}{2}}^{(1)}$, we could use the WENO interpolation procedure on each component of $u$ as in the scalar case. However, for strong shocks this procedure may lead to oscillatory results. Interpolation in the local characteristic fields, even though more costly, may effectively eliminate such spurious oscillations. The procedure of interpolation in the local characteristic fields can be summarized as follows. At each point $x_{i+\frac{1}{2}}$ where the computation of the numerical flux is needed, we perform the following steps.

1. Compute an average state $u_{i+\frac{1}{2}}$, using either the simple arithmetic mean

$$
u_{i+\frac{1}{2}}=\frac{1}{2}\left(u_{i}+u_{i+1}\right)
$$

or a Roe average [9] satisfying

$$
f\left(u_{i+1}\right)-f\left(u_{i}\right)=f^{\prime}\left(u_{i+\frac{1}{2}}\right)\left(u_{i+1}-u_{i}\right)
$$

if it is available. 
2. Compute the right eigenvectors and the left eigenvectors of the Jacobian $f^{\prime}\left(u_{i+\frac{1}{2}}\right)$, and denote them by

$$
R=R\left(u_{i+\frac{1}{2}}\right), \quad R^{-1}=R^{-1}\left(u_{i+\frac{1}{2}}\right)
$$

3. Transform all the point values which are in the potential stencil of the WENO interpolation for the obtaining $u_{i+\frac{1}{2}}^{ \pm}$, to the local characteristic fields. For our fifth order scheme, we need to compute

$$
v_{j}=R^{-1} u_{j}, \quad j=i-2, \ldots, i+3
$$

4. Perform the scalar WENO interpolation procedure for each component of the characteristic variable $v$, to obtain the corresponding component of the interpolation $v_{i+\frac{1}{2}}^{ \pm}$

5. Transform back into physical space

$$
u_{i+\frac{1}{2}}^{ \pm}=R v_{i+\frac{1}{2}}^{ \pm}
$$

For the two-argument flux $h\left(u^{-}, u^{+}\right)$, instead of the monotone fluxes for the scalar case, we can choose from a variety of numerical fluxes based on exact or approximate Riemann solvers [15]. For example, we can use the Godunov flux, the Lax-Friedrichs flux, the HLLC flux, etc. This is one of the advantages of the alternative formulation discussed in this paper.

For all remaining terms in the Lax-Wendroff process, we can use simple interpolation polynomials based on the same stencil $\left\{x_{i-2}, \ldots, x_{i+3}\right\}$ component-wise to obtain approximations to the values of $u, u_{x}, u_{x x}, u_{x x x}$ and $u_{x x x x}$ at $x_{i+\frac{1}{2}}$. No characteristic decomposition or WENO is needed. We remark that in the algebraic manipulations of the Lax-Wendroff process, the derivatives $f^{\prime}(u), f^{\prime \prime}(u), f^{\prime \prime \prime}(u)$ etc. will become matrices and higher order tensors, increasing the algebraic complexity of the final formulas. 


\subsection{Two-dimensional cases}

Consider the two-dimensional conservation laws:

$$
\left\{\begin{array}{l}
u_{t}+f(u)_{x}+g(u)_{y}=0, \\
u(x, y, 0)=u_{0}(x, y) .
\end{array}\right.
$$

The same Taylor expansion still gives

$$
u(x, y, t+\Delta t)=u(x, y, t)+\Delta t u^{\prime}+\frac{\Delta t^{2}}{2} u^{\prime \prime}+\frac{\Delta t^{3}}{6} u^{\prime \prime \prime}+\frac{\Delta t^{4}}{24} u^{(4)}+\cdots
$$

and we would still like to use the PDE to replace time derivatives by spatial derivatives. The first time derivative $u^{\prime}=-f(u)_{x}-g(u)_{y}$ can be approximated in a dimension-bydimension fashion as in standard finite difference schemes [13]. For example, for the fifth order case, we have

$$
\begin{aligned}
\hat{f}_{i+\frac{1}{2}, j}^{(1)}= & h_{1}\left(u_{i+\frac{1}{2}, j}^{-}, u_{i+\frac{1}{2}, j}^{+}\right)-\left.\frac{\Delta x^{2}}{24}\left(f^{\prime \prime}(u)\left(u_{x}\right)^{2}+f^{\prime}(u) u_{x x}\right)\right|_{\left(x_{i+\frac{1}{2}}, y_{j}\right)}+\frac{7 \Delta x^{4}}{5760}\left(f^{\prime \prime \prime \prime}(u)\left(u_{x}\right)^{4}\right. \\
& \left.+6 f^{\prime \prime \prime}(u)\left(u_{x}\right)^{2} u_{x x}+3 f^{\prime \prime}(u)\left(u_{x x}\right)^{2}+4 f^{\prime \prime}(u) u_{x} u_{x x x}+f^{\prime}(u) u_{x x x x}\right)\left.\right|_{\left(x_{i+\frac{1}{2}}, y_{j}\right)} \\
\hat{g}_{i, j+\frac{1}{2}}^{(1)}= & h_{2}\left(u_{i, j+\frac{1}{2}}^{-}, u_{i, j+\frac{1}{2}}^{+}\right)-\left.\frac{\Delta y^{2}}{24}\left(g^{\prime \prime}(u)\left(u_{y}\right)^{2}+g^{\prime}(u) u_{y y}\right)\right|_{\left(x_{i}, y_{j+\frac{1}{2}}\right.}+\frac{7 \Delta y^{4}}{5760}\left(g^{\prime \prime \prime \prime}(u)\left(u_{y}\right)^{4}\right. \\
& \left.+6 g^{\prime \prime \prime}(u)\left(u_{y}\right)^{2} u_{y y}+3 g^{\prime \prime}(u)\left(u_{y y}\right)^{2}+4 g^{\prime \prime}(u) u_{y} u_{y y y}+g^{\prime}(u) u_{y y y y}\right)\left.\right|_{\left(x_{i}, y_{j+\frac{1}{2}}\right)}
\end{aligned}
$$

such that

$$
\begin{aligned}
& \frac{\hat{f}_{i+\frac{1}{2}, j}^{(1)}-\hat{f}_{i-\frac{1}{2}, j}^{(1)}}{\Delta x}=\left.f(u)_{x}\right|_{\left(x_{i}, y_{j}\right)}+O\left(\Delta x^{5}\right), \\
& \frac{\hat{g}_{i, j+\frac{1}{2}}^{(1)}-\hat{g}_{i, j-\frac{1}{2}}^{(1)}}{\Delta y}=\left.g(u)_{y}\right|_{\left(x_{i}, y_{j}\right)}+O\left(\Delta y^{5}\right),
\end{aligned}
$$

and the values of $u_{i+\frac{1}{2}, j}^{ \pm}, u_{i, j+\frac{1}{2}}^{ \pm}$in the monotone fluxes $h_{1}(\cdot, \cdot)$ (approximating $f(u)$ ) and $h_{2}(\cdot, \cdot)$ (approximating $\left.g(u)\right)$ are obtained by one-dimensional WENO interpolation in the same procedure as in the one-dimensional case. The values of $u$ and all its spatial derivatives in all the remaining terms are approximated by simple one-dimensional polynomial interpolations on the same stencil as the WENO approximation, namely on $S=\left\{x_{i-2}, \ldots, x_{i+3}\right\}$ for fixed $y_{j}$ or on $\left\{y_{j-2}, \ldots, y_{j+3}\right\}$ for fixed $x_{i}$. This part is also the same as in the one-dimensional case. 
For the second time derivative, $u^{\prime \prime}=\left(f^{\prime}(u)^{2} u_{x}+f^{\prime}(u) g^{\prime}(u) u_{y}\right)_{x}+\left(g^{\prime}(u) f^{\prime}(u) u_{x}+\right.$ $\left.g^{\prime}(u)^{2} u_{y}\right)_{y}$, we can also approximate them in a dimension-by-dimension fashion. For the fifth order scheme, the numerical fluxes have the following form:

$$
\begin{aligned}
\hat{f}_{i+\frac{1}{2}, j}^{(2)}= & -\left.\left(f^{\prime}(u)^{2} u_{x}+f^{\prime}(u) g^{\prime}(u) u_{y}\right)\right|_{\left(x_{i+\frac{1}{2},}, y_{j}\right)}+\frac{\Delta x^{2}}{24}\left(2 f^{\prime}(u) f^{\prime \prime \prime}(u)\left(u_{x}\right)^{3}+2\left(f^{\prime \prime}(u)\right)^{2}\left(u_{x}\right)^{3}\right. \\
& +6 f^{\prime}(u) f^{\prime \prime}(u) u_{x} u_{x x}+\left(f^{\prime}(u)\right)^{2} u_{x x x}+f^{\prime \prime \prime}(u) g^{\prime}(u)\left(u_{x}\right)^{2} u_{y}+2 f^{\prime \prime}(u) g^{\prime \prime}(u)\left(u_{x}\right)^{2} u_{y} \\
& +f^{\prime}(u) g^{\prime \prime \prime}(u)\left(u_{x}\right)^{2} u_{y}+f^{\prime \prime}(u) g^{\prime}(u) u_{x x} u_{y}+f^{\prime}(u) g^{\prime \prime}(u) u_{x x} u_{y} \\
& \left.+2 f^{\prime \prime}(u) g^{\prime}(u) u_{x} u_{x y}+2 f^{\prime}(u) g^{\prime \prime}(u) u_{x} u_{x y}+f^{\prime}(u) g^{\prime}(u) u_{x x y}\right)\left.\right|_{\left(x_{i+\frac{1}{2}}, y_{j}\right)} \\
\hat{g}_{i, j+\frac{1}{2}}^{(2)}= & -\left.\left(g^{\prime}(u) f^{\prime}(u) u_{x}+\left(g^{\prime}(u)\right)^{2} u_{y}\right)\right|_{\left(x_{i}, y_{j+\frac{1}{2}}\right)}+\frac{\Delta y^{2}}{24}\left(2 g^{\prime}(u) g^{\prime \prime \prime}(u)\left(u_{y}\right)^{3}+2\left(g^{\prime \prime}(u)\right)^{2}\left(u_{y}\right)^{3}\right. \\
& +6 g^{\prime}(u) g^{\prime \prime}(u) u_{y} u_{y y}+\left(g^{\prime}(u)\right)^{2} u_{y y y}+g^{\prime \prime \prime}(u) f^{\prime}(u) u_{x}\left(u_{y}\right)^{2}+2 g^{\prime \prime}(u) f^{\prime \prime}(u) u_{x}\left(u_{y}\right)^{2} \\
& +g^{\prime}(u) f^{\prime \prime \prime}(u) u_{x}\left(u_{y}\right)^{2}+g^{\prime \prime}(u) f^{\prime}(u) u_{x} u_{y y}+g^{\prime}(u) f^{\prime \prime}(u) u_{x} u_{y y} \\
& \left.+2 g^{\prime \prime}(u) f^{\prime}(u) u_{y} u_{x y}+2 g^{\prime}(u) f^{\prime \prime}(u) u_{y} u_{x y}+g^{\prime}(u) f^{\prime}(u) u_{x y y}\right)\left.\right|_{\left(x_{i}, y_{j+\frac{1}{2}}\right)}
\end{aligned}
$$

which satisfies

$$
\begin{aligned}
& \frac{\hat{f}_{i+\frac{1}{2}, j}^{(2)}-\hat{f}_{i-\frac{1}{2}, j}^{(2)}}{\Delta x}=-\left.\left(f^{\prime}(u)^{2} u_{x}+f^{\prime}(u) g^{\prime}(u) u_{y}\right)_{x}\right|_{\left(x_{i}, y_{j}\right)}+O\left(\Delta x^{4}\right)+O\left(\Delta y^{4}\right) \\
& \frac{\hat{g}_{i, j+\frac{1}{2}}^{(2)}-\hat{g}_{i, j-\frac{1}{2}}^{(2)}}{\Delta y}=-\left.\left(g^{\prime}(u) f^{\prime}(u) u_{x}+g^{\prime}(u)^{2} u_{y}\right)_{y}\right|_{\left(x_{i}, y_{j}\right)}+O\left(\Delta x^{4}\right)+O\left(\Delta y^{4}\right) .
\end{aligned}
$$

All the spatial derivatives can be obtained by using simple one- or two-dimensional polynomial interpolations on the same stencil as the WENO approximation. Twodimensional interpolation on the stencil $S=\left\{x_{i-2}, \ldots, x_{i+3}\right\} \times\left\{y_{j-2}, \ldots, y_{j+3}\right\}$ is needed to obtain approximations of the mixed derivatives. This part is different from the onedimensional case. The higher order time derivatives can be approximated in a similar way.

Once the fluxes for all the time derivatives have been obtained, we can form the final numerical flux as the sum of all these fluxes

$$
\begin{aligned}
& \hat{f}_{i+\frac{1}{2}, j}=\hat{f}_{i+\frac{1}{2}, j}^{(1)}+\frac{\Delta t}{2} \hat{f}_{i+\frac{1}{2}, j}^{(2)}+\frac{\Delta t^{2}}{6} \hat{f}_{i+\frac{1}{2}, j}^{(3)}+\frac{\Delta t^{3}}{24} \hat{f}_{i+\frac{1}{2}, j}^{(4)} \\
& \hat{g}_{i, j+\frac{1}{2}}=\hat{g}_{i, j+\frac{1}{2}}^{(1)}+\frac{\Delta t}{2} \hat{g}_{i, j+\frac{1}{2}}^{(2)}+\frac{\Delta t^{2}}{6} \hat{g}_{i, j+\frac{1}{2}}^{(3)}+\frac{\Delta t^{3}}{24} \hat{g}_{i, j+\frac{1}{2}}^{(4)}
\end{aligned}
$$


and obtain the final scheme

$$
u_{i, j}^{n+1}=u_{i, j}^{n}-\frac{\Delta t}{\Delta x}\left(\hat{f}_{i+\frac{1}{2}, j}-\hat{f}_{i-\frac{1}{2}, j}\right)-\frac{\Delta t}{\Delta y}\left(\hat{g}_{i, j+\frac{1}{2}}-\hat{g}_{i, j-\frac{1}{2}}\right)
$$

which is fifth order accurate in space and fourth order accurate in time.

For the system case, WENO interpolation with local characteristic decomposition is still needed for the computation of $u_{i+\frac{1}{2}, j}^{ \pm}$and $u_{i, j+\frac{1}{2}}^{ \pm}$in the two-point fluxes $h_{1}(\cdot, \cdot)$ and $h_{2}(\cdot, \cdot)$. This part is the same as in the one-dimensional case and uses the information of the Jacobians $f^{\prime}(u)$ and $g^{\prime}(u)$ in the $x$ and $y$ directions respectively. For all the other terms, neither local characteristic decomposition nor WENO interpolation is needed. We can use the interpolation polynomials as described above for the scalar case in each component of the solution $u$.

\section{Numerical results}

In this section, we present the results of our numerical experiments for the schemes described in the previous section, with fourth order in temporal and fifth order in spatial accuracy. In some examples we also compare with the Lax-Wendroff type finite difference WENO scheme in [8], also with fourth order in temporal and fifth order in spatial accuracy.

Example 1. We first test the accuracy of the scheme on the linear scalar problem

$$
u_{t}+u_{x}=0
$$

with the initial condition $u(x, 0)=\sin (\pi x)$ and 2-periodic boundary condition. The exact solution is $u(x, t)=\sin (\pi(x-t))$. In Table 1 , we show the errors at time $t=2$. We can observe fifth order accuracy until quite refined meshes, despite of the formal fourth order temporal accuracy, supporting our claim before that spatial errors are usually dominating for modest meshes. 
Table 1: Accuracy on $u_{t}+u_{x}=0$ with $u(x, 0)=\sin (\pi x)$ at $t=2$.

\begin{tabular}{|c|c|c|c|c|}
\hline$N_{x}$ & $L_{1}$ errors & order & $L_{\infty}$ error & order \\
\hline 10 & $2.38 \mathrm{E}-02$ & - & $3.67 \mathrm{E}-02$ & - \\
20 & $1.12 \mathrm{E}-03$ & 4.41 & $1.99 \mathrm{E}-03$ & 4.20 \\
40 & $3.45 \mathrm{E}-05$ & 5.02 & $6.64 \mathrm{E}-05$ & 4.91 \\
80 & $1.07 \mathrm{E}-06$ & 5.00 & $2.16 \mathrm{E}-06$ & 4.94 \\
160 & $3.35 \mathrm{E}-08$ & 5.00 & $6.51 \mathrm{E}-08$ & 5.05 \\
320 & $1.05 \mathrm{E}-09$ & 5.00 & $1.95 \mathrm{E}-09$ & 5.06 \\
640 & $3.25 \mathrm{E}-11$ & 5.01 & $5.71 \mathrm{E}-11$ & 5.10 \\
\hline
\end{tabular}

Example 2. We test our scheme on the nonlinear Burgers equation

$$
u_{t}+\left(\frac{u^{2}}{2}\right)_{x}=0
$$

with the initial condition $u(x, 0)=0.5+\sin (\pi x)$ and a 2-periodic boundary condition. All the three monotone fluxes mentioned in Section 3.1, namely the Godunov flux, the Engquist-Osher flux and the Lax-Friedrichs flux, are tested.

When $t=0.5 / \pi$ the solution is still smooth. The errors for all three monotone fluxes are shown in Tables 2 and 3 respectively. For comparison, we also show the errors by the Lax-Wendroff type finite difference WENO scheme in [8] with the same fourth order in temporal and fifth order in spatial accuracy in Table 2. We observe that all schemes achieve fifth order accuracy, despite of the formal fourth order temporal accuracy, once again supporting our claim before that spatial errors are usually dominating for modest meshes. For this test case, there is very little difference in the errors corresponding to the three different monotone fluxes. We do observe that the errors of the WENO scheme in [8] are significantly bigger than those for the schemes designed in this paper in Tables 2 and 3 when compared on the same mesh, indicating that the narrower effective stencil and flexibility in choosing numerical fluxes are helping to improve the magnitude of the errors.

We plot the solution with 80 grid points at time $t=1.5 / \pi$ in Figure 1 , when a shock has already appeared in the solution. We can see that for all the three monotone fluxes, the schemes simulate the solution with good resolution and without noticeable 
oscillations near the shock.

Table 2: Accuracy on the Burgers equation with the Lax-Friedrichs flux and the WENO scheme in [8]. $u(x, 0)=0.5+\sin (\pi x)$ at $t=0.5 / \pi$.

\begin{tabular}{|c|c|c|c|c|c|c|c|c|}
\hline & \multicolumn{4}{|c|}{ Lax-Friedrichs flux } & \multicolumn{4}{c|}{ WENO scheme in $[8]$} \\
\hline$N_{x}$ & $L_{1}$ errors & order & $L_{\infty}$ error & order & $L_{1}$ errors & order & $L_{\infty}$ error & order \\
\hline 10 & $4.57 \mathrm{E}-03$ & - & $1.42 \mathrm{E}-02$ & - & $3.65 \mathrm{E}-02$ & - & $6.85 \mathrm{E}-02$ & - \\
20 & $4.85 \mathrm{E}-04$ & 3.24 & $2.33 \mathrm{E}-03$ & 2.61 & $4.44 \mathrm{E}-03$ & 3.04 & $1.26 \mathrm{E}-02$ & 2.45 \\
40 & $2.59 \mathrm{E}-05$ & 4.23 & $2.38 \mathrm{E}-04$ & 3.29 & $2.55 \mathrm{E}-04$ & 4.12 & $1.06 \mathrm{E}-03$ & 3.57 \\
80 & $1.37 \mathrm{E}-06$ & 4.23 & $1.14 \mathrm{E}-05$ & 4.38 & $9.99 \mathrm{E}-06$ & 4.67 & $5.11 \mathrm{E}-05$ & 4.38 \\
160 & $5.97 \mathrm{E}-08$ & 4.52 & $9.90 \mathrm{E}-07$ & 3.53 & $3.76 \mathrm{E}-07$ & 4.73 & $1.69 \mathrm{E}-06$ & 4.92 \\
320 & $1.80 \mathrm{E}-09$ & 5.05 & $3.69 \mathrm{E}-08$ & 4.74 & $1.15 \mathrm{E}-08$ & 5.03 & $7.62 \mathrm{E}-08$ & 4.47 \\
640 & $4.07 \mathrm{E}-11$ & 5.47 & $3.97 \mathrm{E}-10$ & 6.54 & $2.96 \mathrm{E}-10$ & 5.28 & $1.46 \mathrm{E}-09$ & 5.70 \\
\hline
\end{tabular}

Table 3: Accuracy on the Burgers equation with the Godunov flux and Engquist-Osher flux. $u(x, 0)=0.5+\sin (\pi x)$ at $t=0.5 / \pi$.

\begin{tabular}{|c|c|c|c|c|c|c|c|c|}
\hline & \multicolumn{4}{|c|}{ Godunov flux } & \multicolumn{4}{c|}{ Engquist-Osher flux } \\
\hline$N_{x}$ & $L_{1}$ errors & order & $L_{\infty}$ error & order & $L_{1}$ errors & order & $L_{\infty}$ error & order \\
\hline 10 & $4.59 \mathrm{E}-03$ & - & $1.41 \mathrm{E}-02$ & - & $4.59 \mathrm{E}-03$ & - & $1.41 \mathrm{E}-02$ & - \\
20 & $4.84 \mathrm{E}-04$ & 3.24 & $2.32 \mathrm{E}-03$ & 2.60 & $4.84 \mathrm{E}-04$ & 3.24 & $2.32 \mathrm{E}-03$ & 2.60 \\
40 & $2.59 \mathrm{E}-05$ & 4.23 & $2.38 \mathrm{E}-04$ & 3.29 & $2.59 \mathrm{E}-05$ & 4.23 & $2.38 \mathrm{E}-04$ & 3.29 \\
80 & $1.37 \mathrm{E}-06$ & 4.23 & $1.14 \mathrm{E}-05$ & 4.38 & $1.37 \mathrm{E}-06$ & 4.23 & $1.14 \mathrm{E}-05$ & 4.38 \\
160 & $5.97 \mathrm{E}-08$ & 4.52 & $9.90 \mathrm{E}-07$ & 3.53 & $5.97 \mathrm{E}-08$ & 4.52 & $9.90 \mathrm{E}-07$ & 3.53 \\
320 & $1.80 \mathrm{E}-09$ & 5.05 & $3.69 \mathrm{E}-08$ & 4.74 & $1.80 \mathrm{E}-09$ & 5.05 & $3.69 \mathrm{E}-08$ & 4.74 \\
640 & $4.07 \mathrm{E}-11$ & 5.47 & $3.97 \mathrm{E}-10$ & 6.54 & $4.07 \mathrm{E}-11$ & 5.47 & $3.97 \mathrm{E}-10$ & 6.54 \\
\hline
\end{tabular}

Example 3. We now consider the one-dimensional Euler system of compressible gas dynamics:

$$
\xi_{t}+f(\xi)_{x}=0
$$

where

$$
\xi=(\rho, \rho u, E)^{T}, \quad f(\xi)=\left(\rho u, \rho u^{2}+p, u(E+p)\right)^{T} .
$$

Here $\rho$ is the density, $u$ is the velocity, $E$ is the total energy, and $p$ is the pressure, which is related to the total energy by $E=\frac{p}{\gamma-1}+\frac{1}{2} \rho u^{2}$ with $\gamma=1.4$ for air. The initial condition is set to be $\rho(x, 0)=1+0.2 \sin (\pi x), u(x, 0)=0.7, p(x, 0)=1$, with a 2 -periodic 


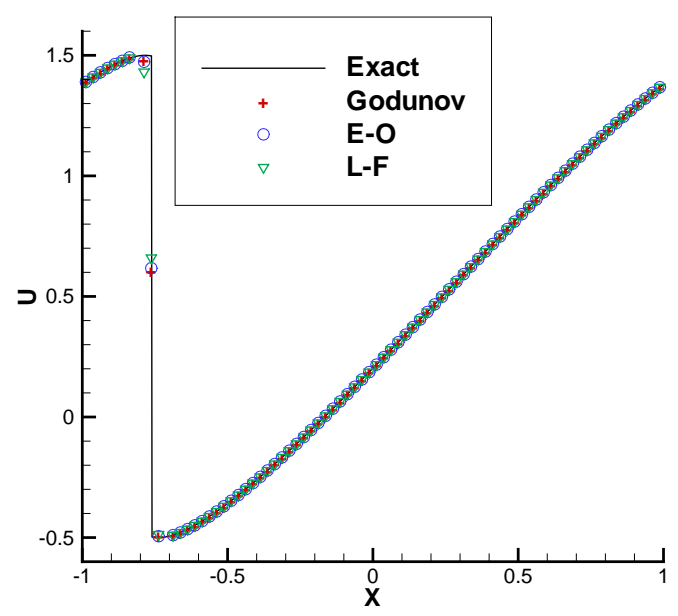

Figure 1: The Burgers equation with $u(x, 0)=0.5+\sin (\pi x) . t=1.5 / \pi . \quad N=80$ grid points.

boundary condition. The exact solution is $\rho(x, t)=1+0.2 \sin (\pi(x-u t)), u(x, t)=0.7$ and $p(x, t)=1$. The errors and numerical orders of accuracy for the density $\rho$ are shown in Table 4. We can see that the schemes with with both the Lax-Friedrichs flux and the HLLC flux can achieve the designed order of accuracy, while the error magnitude is smaller for the HLLC flux than for the Lax-Friedrichs flux on the same mesh.

Table 4: Errors and orders of accuracy for the density $\rho$ on the one-dimension Euler equation at $t=2$. Lax-Friedrichs flux and HLLC flux.

\begin{tabular}{|c|c|c|c|c|c|c|c|c|}
\hline & \multicolumn{4}{|c|}{ Lax-Friedrichs flux } & \multicolumn{4}{c|}{ HLLC flux } \\
\hline$N_{x}$ & $L_{1}$ errors & order & $L_{\infty}$ error & order & $L_{1}$ errors & order & $L_{\infty}$ error & order \\
\hline 10 & $9.20 \mathrm{E}-03$ & - & $1.30 \mathrm{E}-02$ & - & $3.46 \mathrm{E}-03$ & - & $5.58 \mathrm{E}-03$ & - \\
20 & $4.84 \mathrm{E}-04$ & 4.25 & $7.74 \mathrm{E}-04$ & 4.07 & $1.55 \mathrm{E}-04$ & 4.48 & $2.93 \mathrm{E}-04$ & 4.25 \\
40 & $1.53 \mathrm{E}-05$ & 4.98 & $2.80 \mathrm{E}-05$ & 4.79 & $4.82 \mathrm{E}-06$ & 5.01 & $1.00 \mathrm{E}-05$ & 4.87 \\
80 & $4.77 \mathrm{E}-07$ & 5.00 & $8.89 \mathrm{E}-07$ & 4.98 & $1.50 \mathrm{E}-07$ & 5.01 & $3.06 \mathrm{E}-07$ & 5.04 \\
160 & $1.47 \mathrm{E}-08$ & 5.02 & $2.67 \mathrm{E}-08$ & 5.06 & $4.63 \mathrm{E}-09$ & 5.02 & $8.38 \mathrm{E}-09$ & 5.19 \\
320 & $4.42 \mathrm{E}-10$ & 5.06 & $7.52 \mathrm{E}-10$ & 5.15 & $1.39 \mathrm{E}-10$ & 5.06 & $2.37 \mathrm{E}-10$ & 5.14 \\
640 & $1.19 \mathrm{E}-11$ & 5.21 & $1.99 \mathrm{E}-11$ & 5.24 & $3.72 \mathrm{E}-12$ & 5.22 & $6.44 \mathrm{E}-12$ & 5.20 \\
\hline
\end{tabular}

Example 4. Next, we solve the same one-dimensional Euler equations with a Riemann 
initial condition for the Sod's problem

$$
(\rho, u, p)= \begin{cases}(1,0,1), & \text { if } x \leq 0 \\ (0.125,0,0.1), & \text { if } x>0\end{cases}
$$

and the Lax's problem

$$
(\rho, u, p)= \begin{cases}(0.445,0.698,3.528), & \text { if } x \leq 0 \\ (0.5,0,0.571), & \text { if } x>0\end{cases}
$$

We plot the computed density at $t=1.3$ for both problems with 200 grid points. We can see the results of both problems are well resolved and non-oscillatory with Lax-Friedrichs flux and HLLC flux.

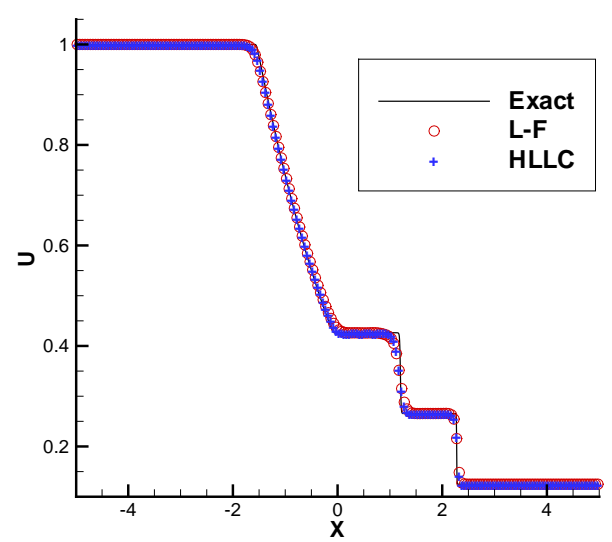

(a) The Sod's problem

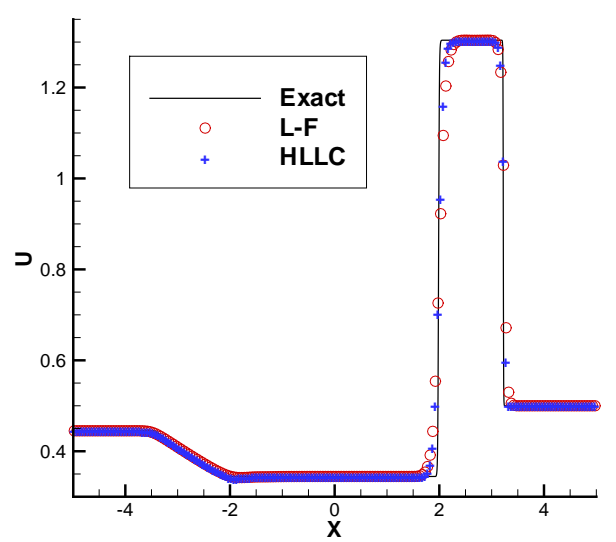

(b) The Lax's problem

Figure 2: The Sod's and Lax's problems. $t=1.3 .200$ grid points with Lax-Friedrichs flux and HLLC flux.

Example 5. We plot the numerical solution of the blast wave problem. We are still solving the Euler Equations (36)-(37), with the initial condition

$$
\begin{gathered}
\rho(x, 0)=1.0, \quad 0 \leq x \leq 1 \\
u(x, 0)=0.0, \quad 0 \leq x \leq 1 \\
p(x, 0)= \begin{cases}1000, & \text { if } \quad 0 \leq x<0.1 \\
0.01, & \text { if } 0.1 \leq x<0.9 \\
100, & \text { if } \quad 0.9 \leq x \leq 1 .\end{cases}
\end{gathered}
$$


We compare numerical solution with a reference solution, which is obtained with the regular WENO scheme in [4] with 16000 grid points and hence can be considered as the exact solution. We use both Lax-Friedrichs flux and HLLC flux for the problem. 800 grid points are used. Our numerical results seem to agree with the reference solution very well.

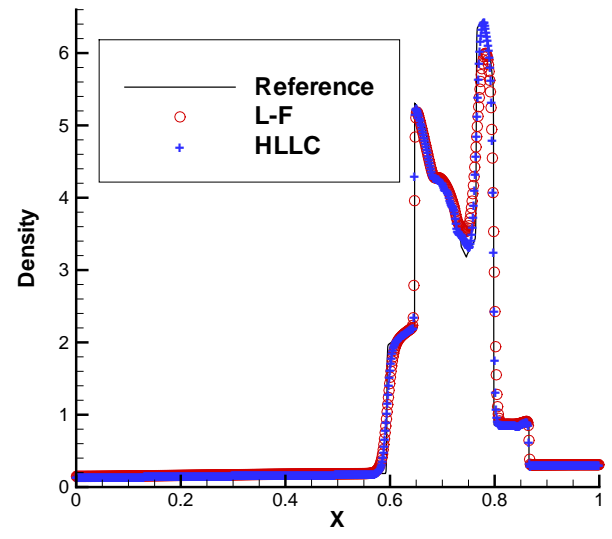

(a) Density

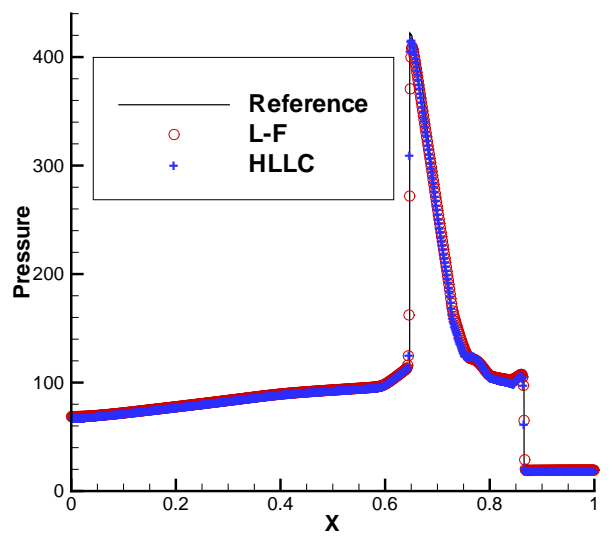

(b) Pressure

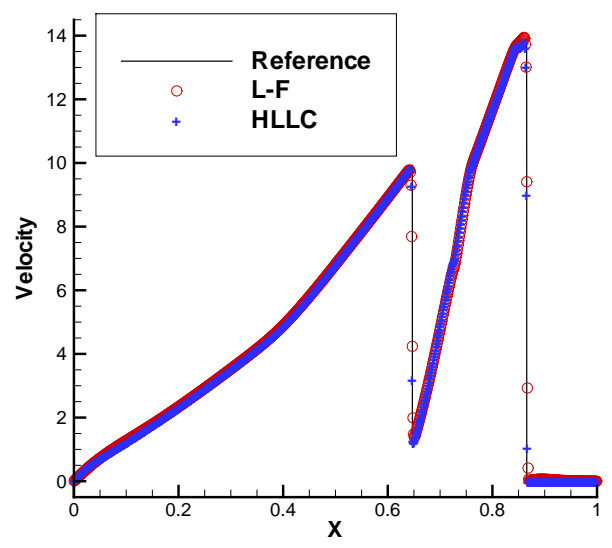

(c) Velocity

Figure 3: Blast wave problem. Lines are from the reference solution. Circles are the numerical solution with Lax-Friedrichs flux, and pluses are the numerical with HLLC flux: (a) density; (b) pressure; (c) velocity.

Example 6. We now consider the following two-dimensional linear equation

$$
u_{t}+a u_{x}+b u_{y}=0
$$


with the initial condition $u(x, y, 0)=\sin (\pi(x+y))$ and a 2-periodic boundary condition, where $a$ and $b$ are constants. Here, we take $a=1, b=-2$, and the final time $t=2$. To avoid possible error cancellations due to symmetry, we use different mesh sizes in the $x$ and $y$ directions. Errors are shown in Table 5. We can clearly observe that the scheme achieves the designed fifth order accuracy.

Table 5: Accuracy on the linear equation $u_{t}+u_{x}-2 u_{y}=0$ with $u(x, y, 0)=\sin (\pi(x+y))$ at $t=2$.

\begin{tabular}{|c|c|c|c|c|}
\hline$N_{x} \times N_{y}$ & $L_{1}$ errors & order & $L_{\infty}$ error & order \\
\hline $8 \times 12$ & $4.97 \mathrm{E}-02$ & - & $7.23 \mathrm{E}-02$ & - \\
$16 \times 24$ & $5.70 \mathrm{E}-03$ & 3.12 & $1.02 \mathrm{E}-02$ & 2.83 \\
$32 \times 48$ & $1.70 \mathrm{E}-04$ & 5.07 & $3.42 \mathrm{E}-04$ & 4.90 \\
$64 \times 96$ & $4.72 \mathrm{E}-06$ & 5.17 & $1.01 \mathrm{E}-05$ & 5.08 \\
$128 \times 192$ & $1.41 \mathrm{E}-07$ & 5.07 & $3.07 \mathrm{E}-07$ & 5.04 \\
$256 \times 384$ & $4.32 \mathrm{E}-09$ & 5.02 & $9.16 \mathrm{E}-09$ & 5.06 \\
\hline
\end{tabular}

Example 7. Next, we solve the two-dimensional nonlinear Burgers equation

$$
u_{t}+\left(\frac{u^{2}}{2}\right)_{x}+\left(\frac{u^{2}}{2}\right)_{y}=0
$$

with the initial condition $u(x, 0)=0.5+\sin (\pi(x+y) / 2)$ and a 4-periodic boundary condition. When $t=0.5 / \pi$ the solution is still smooth, and we give the errors and order of accuracy in Table 6 with the Lax-Friedrichs flux. Clearly, the scheme delivers the designed order of accuracy. For comparison, we also show the errors by the Lax-Wendroff type finite difference WENO scheme in [8] with the same fourth order in temporal and fifth order in spatial accuracy in Table 6. We again observe that the errors of the WENO scheme in [8] are significantly bigger than those for the schemes designed in this paper in Table 6 when compared on the same mesh, indicating that the narrower effective stencil is helping in improving the magnitude of the errors.

We plot the results at $t=1.5 / \pi$ when shocks have already appeared in Figure 4 with $160 \times 160$ grid points. On the left we are showing a one-dimensional cut at $x=y$ of the solution, and on the right we are showing the surface of the computed solution. Clearly, the shocks are well resolved without spurious oscillations. 
Table 6: Accuracy on the Burgers equation $u_{t}+\left(\frac{u^{2}}{2}\right)_{x}+\left(\frac{u^{2}}{2}\right)_{y}=0$ with Lax-Friedrichs flux and the WENO scheme in [8]. $u(x, y, 0)=0.5+\sin (\pi(x+y) / 2)$ at $t=0.5 / \pi$.

\begin{tabular}{|c|c|c|c|c|c|c|c|c|}
\hline & \multicolumn{4}{|c|}{ Lax-Friedrichs flux } & \multicolumn{4}{c|}{ WENO scheme in $[8]$} \\
\hline$N_{x} \times N_{y}$ & $L_{1}$ errors & order & $L_{\infty}$ error & order & $L_{1}$ errors & order & $L_{\infty}$ error & order \\
\hline $8 \times 12$ & $7.51 \mathrm{E}-03$ & - & $2.35 \mathrm{E}-02$ & - & $2.23 \mathrm{E}-02$ & - & $6.44 \mathrm{E}-02$ & - \\
$16 \times 24$ & $9.86 \mathrm{E}-04$ & 2.93 & $6.96 \mathrm{E}-03$ & 1.75 & $2.98 \mathrm{E}-03$ & 2.90 & $1.45 \mathrm{E}-02$ & 2.15 \\
$32 \times 48$ & $8.23 \mathrm{E}-05$ & 3.58 & $7.30 \mathrm{E}-04$ & 3.25 & $2.90 \mathrm{E}-04$ & 3.36 & $1.65 \mathrm{E}-03$ & 3.13 \\
$64 \times 96$ & $4.26 \mathrm{E}-06$ & 4.27 & $4.09 \mathrm{E}-05$ & 4.16 & $8.58 \mathrm{E}-06$ & 5.08 & $8.08 \mathrm{E}-05$ & 4.36 \\
$128 \times 192$ & $1.84 \mathrm{E}-07$ & 4.53 & $1.62 \mathrm{E}-06$ & 4.65 & $3.27 \mathrm{E}-07$ & 4.71 & $2.57 \mathrm{E}-06$ & 4.97 \\
$256 \times 384$ & $6.24 \mathrm{E}-09$ & 4.88 & $7.20 \mathrm{E}-08$ & 4.49 & $1.01 \mathrm{E}-08$ & 5.01 & $1.33 \mathrm{E}-07$ & 4.28 \\
\hline
\end{tabular}

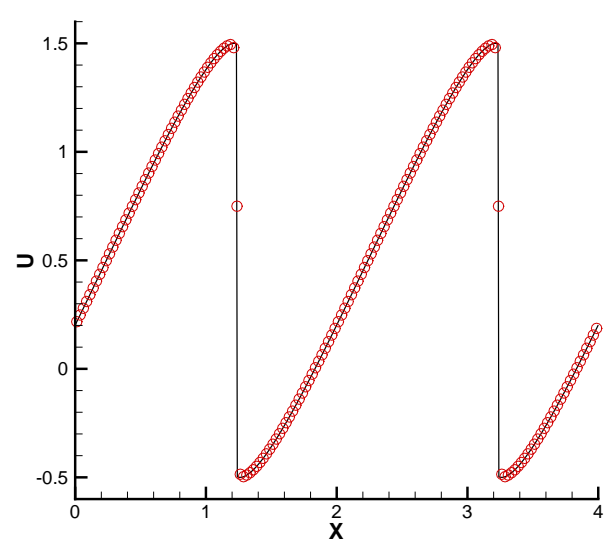

(a)

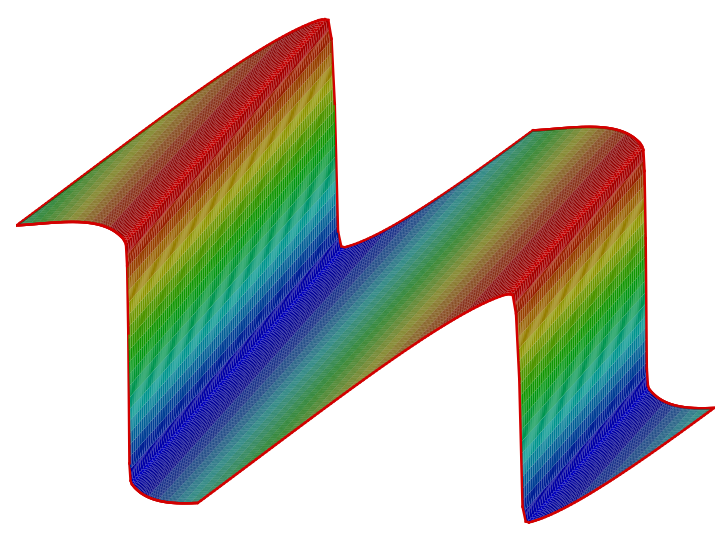

(b)

Figure 4: The two-dimensional Burgers equation. $u(x, 0)=0.5+\sin (\pi(x+y) / 2) . t=$

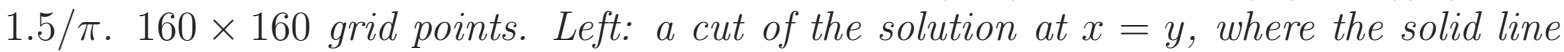
is the exact solution and the circles are the computed solution; right: the surface of the solution.

Example 8. We now consider two-dimensional Euler systems of compressible gas dynamics:

$$
\xi_{t}+f(\xi)_{x}+g(\xi)_{y}=0
$$


with

$$
\begin{aligned}
\xi & =(\rho, \rho u, \rho v, E)^{T}, \\
f(\xi) & =\left(\rho u, \rho u^{2}+p, \rho u v, u(E+p)\right)^{T}, \\
g(\xi) & =\left(\rho v, \rho u v, \rho v^{2}+p, v(E+p)\right)^{T} .
\end{aligned}
$$

Here, $\rho$ is the density, $(u, v)$ is velocity, $E$ is the total energy, and $p$ is the pressure, related to the total energy $E$ by $E=\frac{p}{\gamma-1}+\frac{1}{2} \rho\left(u^{2}+v^{2}\right)$, with $\gamma=1.4$. The initial condition is set to be $\rho(x, y, 0)=1+0.2 \sin (\pi(x+y)), u(x, y, 0)=0.7, v(x, y, 0)=0.3$, $p(x, y, 0)=1$, with a 2 -periodic boundary condition. The exact solution is $\rho(x, y, t)=$ $1+0.2 \sin (\pi(x+y-(u+v) t)), u=0.7, v=0.3, p=1$. The errors and order of accuracy for the density are shown in Table 7 . We can see that the scheme achieves the designed order of accuracy.

Table 7: Accuracy on the two-dimensional Euler equation at $t=2$. Errors of the density $\rho$.

\begin{tabular}{|c|c|c|c|c|}
\hline$N_{x} \times N_{y}$ & $L_{1}$ errors & order & $L_{\infty}$ error & order \\
\hline $8 \times 12$ & $2.29 \mathrm{E}-02$ & - & $3.28 \mathrm{E}-02$ & - \\
$16 \times 24$ & $1.47 \mathrm{E}-03$ & 3.97 & $2.22 \mathrm{E}-03$ & 3.89 \\
$32 \times 48$ & $5.15 \mathrm{E}-05$ & 4.83 & $9.03 \mathrm{E}-05$ & 4.62 \\
$64 \times 96$ & $1.61 \mathrm{E}-06$ & 5.00 & $2.99 \mathrm{E}-06$ & 4.92 \\
$128 \times 192$ & $4.98 \mathrm{E}-08$ & 5.01 & $9.20 \mathrm{E}-08$ & 5.02 \\
$256 \times 384$ & $1.51 \mathrm{E}-09$ & 5.04 & $2.58 \mathrm{E}-09$ & 5.15 \\
\hline
\end{tabular}

Example 9. Next, we use the following problem to illustrate further the high order accuracy of the method. Consider the following idealized problem for the Euler equation in 2D: The mean flow is $\rho=1, p=1$, and $(u, v)=(1,1)$ (diagonal flow). We add, to this mean flow, an isentropic vortex (perturbation in $(u, v)$ and the temperature $T=\frac{p}{\rho}$, no perturbation in the entropy $\left.S=\frac{p}{\rho^{\gamma}}\right)$ :

$$
\begin{aligned}
(\delta u, \delta v) & =\frac{\epsilon}{2 \pi} e^{0.5\left(1-r^{2}\right)}(-\bar{y}, \bar{x}) \\
\delta T & =-\frac{(\gamma-1) \epsilon^{2}}{8 \gamma \pi^{2}} e^{1-r^{2}} \\
\delta S & =0
\end{aligned}
$$


where $(\bar{x}, \bar{y})=(x-5, y-5), r^{2}=\bar{x}^{2}+\bar{y}^{2}$, and the vortex strength $\epsilon=5$ [11]. Since the mean flow is in the diagonal direction, the vortex movement is not aligned with the mash direction. It is clear the the exact solutions is just the passive convection of the vortex with the mean velocity. The computational domain is taken as $[0,10] \times[0,10]$, extended periodically in both directions. The simulation is performed until $t=0.2$. The errors and orders of accuracy are shown in Table 8.

Table 8: Accuracy on the Vortex evolution at $t=0.2$. Errors of the density $\rho$.

\begin{tabular}{|c|c|c|c|c|}
\hline$N_{x} \times N_{y}$ & $L_{1}$ errors & order & $L_{\infty}$ error & order \\
\hline $40 \times 40$ & $5.52 \mathrm{E}-03$ & 7.50 & $1.98 \mathrm{E}-03$ & 8.98 \\
$80 \times 80$ & $2.90 \mathrm{E}-04$ & 4.25 & $1.41 \mathrm{E}-04$ & 3.81 \\
$160 \times 160$ & $6.84 \mathrm{E}-06$ & 5.41 & $2.57 \mathrm{E}-06$ & 5.78 \\
$320 \times 320$ & $1.96 \mathrm{E}-07$ & 5.13 & $5.53 \mathrm{E}-08$ & 5.54 \\
\hline
\end{tabular}

Example 10. Finally, we consider the double Mach reflection problem. The computational domain for this problem is chosen to be $[0,4] \times[0,1]$. The reflection wall lies at the bottom of the computational domain starting from $x=\frac{1}{6}$. Initially a right-moving Mach 10 shock is positioned at $x=\frac{1}{6}, y=0$ and makes a $60^{\circ}$ angle with the $x$-axis. For the bottom boundary, the exact post-shock condition is imposed for the part from $x=0$ to $x=\frac{1}{6}$ and a reflective boundary condition is used for the rest. At the top boundary of the computational domain, the flow values are set to describe the exact motion of the Mach 10 shock. The initial pre-shock condition is

$$
(\rho, p, u, v)=\left(8,116.5,8.25 \cos \left(30^{\circ}\right),-8.25 \sin \left(30^{\circ}\right)\right)
$$

and the post-shock condition is

$$
(\rho, p, u, v)=(1.4,1,0,0)
$$

We compute the solution up to $t=0.2$. Uniform meshes with grid points $960 \times 239$ are used. In Figure 5 we show 30 equally spaced density contours from 1.5 to 22.7 , and a "zoomed-in" graph is provided in Figure 6. We can see that our results agree well with the results in [8]. 


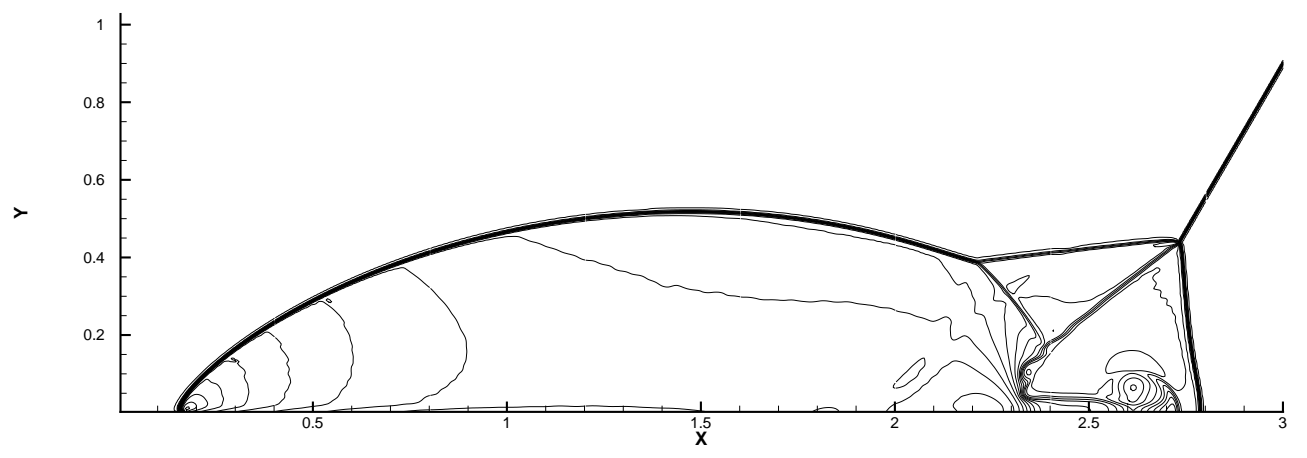

Figure 5: Double Mach reflection problem. 30 equally spaced contours form 1.5 for 22.7 for the density $\rho$.

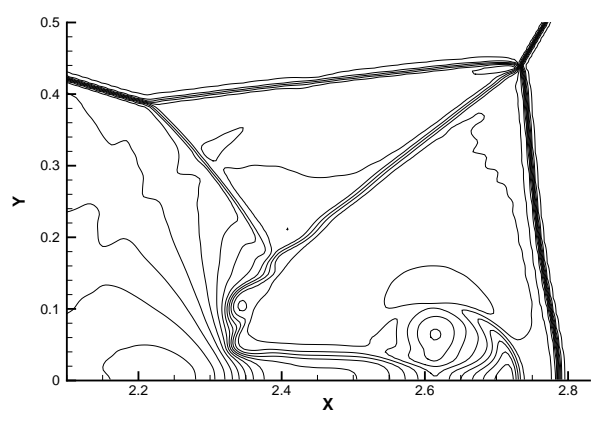

Figure 6: Zoomed-in figure. Double Mach reflection problem. 30 equally spaced contours form 1.5 for 22.7 for the density $\rho$.

\section{Concluding remarks}

We have discussed in depth an alternative formulation for constructing high order conservative finite difference schemes. In this formulation, the high order interpolation procedure is applied to the solution itself rather than to the flux functions. Even though the algebra and algorithm complexity are increased compared with the standard finite difference formulation, this alternative formulation does have several advantages, including the flexibility in using any monotone fluxes in the scalar case and any approximate Riemann solvers in the system case, and the easiness in applying the Lax-Wendroff time discretization, resulting in a scheme with a narrow stencil which is the same as that for 
the semi-discrete scheme. We demonstrate these two advantages, and discuss the second advantage in depth. We describe the Lax-Wendroff time discretization of this alternative formulation of high order conservative finite difference schemes in detail, using fifth order WENO interpolation for the leading term and fixed stencil polynomial interpolation for the remaining terms. Temporal accuracy is kept at fourth order in the extensive numerical results, including one- and two-dimensional scalar problems and systems. These numerical examples verify the performance of the designed schemes in their designed high order of accuracy and non-oscillatory shock transitions for discontinuous solutions. Another advantage of this alternative formulation, namely the easier maintenance of free-stream solutions in multi-dimensional curvilinear coordinates, will be explored in future work.

\section{References}

[1] D. Balsara and C.-W. Shu, Monotonicity preserving weighted essentially nonoscillatory schemes with increasingly high order of accuracy, Journal of Computational Physics, 160 (2000), 405-452.

[2] E. Carlini, R. Ferretti and G. Russo, A weighted essentially nonoscillatory, large time-step scheme for Hamilton-Jacobi equations, SIAM Journal on Scientific Computing, 27 (2005), 1071-1091.

[3] A. Harten, B. Engquist, S. Osher and S. Chakravarthy, Uniformly high order essentially non-oscillatory schemes, III, Journal of Computational Physics, 71 (1987), 231-303.

[4] G. Jiang and C.-W. Shu, Efficient implementation of weighted ENO schemes, Journal of Computational Physics, 126 (1996), 202-228.

[5] P.D. Lax and B. Wendroff, Systems of conservation laws, Communications on Pure and Applied Mathematics, 13 (1960), 217-237. 
[6] R.J. LeVeque, Numerical Methods for Conservation Laws, Birkhauser Verlag, Basel, 1990.

[7] X. Liu, S. Osher and T. Chan, Weighted essentially non-oscillatory schemes, Journal of Computational Physics, 115 (1994), 200-212.

[8] J. Qiu and C.-W. Shu, Finite difference WENO schemes with Lax-Wendroff type time discretizations, SIAM Journal on Scientific Computing, 24 (2003), 2185-2198.

[9] P. Roe, Approximate Riemann solvers, parameter vectors and difference schemes, Journal of Computational Physics, 27 (1978), 1-31.

[10] K. Sebastian and C.-W. Shu, Multi domain WENO finite difference method with interpolation at sub-domain interfaces, Journal of Scientific Computing, 19 (2003), 405-438.

[11] C.-W. Shu, Essentially non-oscillatory and weighted essentially non-oscillatory schemes for hyperbolic conservation laws, in Advanced Numerical Approximation of Nonlinear Hyperbolic Equations, B. Cockburn, C. Johnson, C.-W. Shu and E. Tadmor (Editor: A. Quarteroni), Lecture Notes in Mathematics, volume 1697, Springer, Berlin, 1998, pp.325-432.

[12] C.-W. Shu, High order weighted essentially non-oscillatory schemes for convection dominated problems, SIAM Review, 51 (2009), 82-126.

[13] C.-W. Shu and S. Osher, Efficient implementation of essentially non-oscillatory shock-capturing schemes, Journal of Computational Physics, 77 (1988), 439-471.

[14] C.-W. Shu and S. Osher, Efficient implementation of essentially non-oscillatory shock-capturing schemes, II, Journal of Computational Physics, 83 (1989), 32-78.

[15] E.F. Toro, Riemann Solvers and Numerical Methods for Fluid Dynamics, a Practical Introduction, Springer, Berlin, 1997. 